\title{
Statistical hadronization of charm at SPS, RHIC and LHC
}

\author{
A. Andronic ${ }^{\mathrm{a}}$, P. Braun-Munzinger ${ }^{\mathrm{a}}, \mathrm{K}$. Redlich $^{\mathrm{b}}$, J. Stachel $^{\mathrm{c}}$ \\ ${ }^{a}$ GSI Darmstadt, Germany, ${ }^{\mathrm{b}}$ University of Wroclaw, Poland, ${ }^{\mathrm{c}}$ University of Heidel- \\ berg, Germany
}

We study the production of charmonia and charmed hadrons for nucleus-nucleus collisions at SPS, RHIC, and LHC energies within the framework of the statistical hadronization model. Results from this model are compared to the observed centrality dependence of $J / \psi$ production at SPS energy. We further provide predictions for the centrality dependence of the production of open and hidden charm mesons at RHIC and LHC.

\section{Introduction}

The original idea of statistical hadronization of charm quarks [1,2] has sparked an intense activity in this field [3,4]. Initial interest focussed on the available SPS data on $J / \psi$ production. As we show below, these data can be well described, but only assuming an enhanced charm cross section compared to pQCD calculations. However, the largest differences between results from the statistical coalescence scenario (or a similar model [5]) and more conventional models are expected at collider energies. For example, in the Satz-Matsui approach [6], one would expect very strong suppression compared to direct production of $J / \psi$ mesons (up to a factor of 20 [7]) for central Au-Au collision at RHIC energy. In the present approach this suppression is overcome by statistical recombination of $J / \psi$ mesons from different $c \bar{c}$ pairs, so that much larger yields are expected. We therefore focus in this note on predictions for open and hidden charm mesons at RHIC and LHC energy, with emphasis on the centrality dependence of rapidity densities.

\section{Model description}

The model assumes that all charm quarks are produced in primary hard collisions and equilibrate in quark-gluon plasma (QGP); in particular, no $J / \psi$ is preformed in QGP (complete screening). All charmed hadrons (open and hidden) are formed at freeze-out (at SPS and beyond, freeze-out is at the phase boundary [8]) according to the statistical laws. Taking into account the measured dependence of the ratio $\psi^{\prime} / \psi$ on centrality, the model is valid for not too small number of participants, $N_{\text {part }}$. The total number of thermally produced open charm hadrons, $N_{o c}^{t h}$ is related to the number of directly produced $c \bar{c}$ pairs, $N_{c \bar{c}}^{d i r}$ as (neglecting charmonia): $N_{c \bar{c}}^{d i r}=\frac{1}{2} g_{c} N_{o c}^{t h} I_{1}\left(g_{c} N_{o c}^{t h}\right) / I_{0}\left(g_{c} N_{o c}^{t h}\right)$, from which the charm enhancement factor $g_{c}$ is extracted. $I_{n}$ are the modified Bessel functions. The yield of a given species $X$ is then $N_{X}=g_{c} N_{X}^{t h} I_{1}\left(g_{c} N_{X}^{t h}\right) / I_{0}\left(g_{c} N_{X}^{t h}\right)$ for open charm mesons and hyperons and $N_{X}=g_{c}^{2} N_{X}^{t h}$ for charmonia (see ref. [1,2] for more details). The inputs 
for the above procedure are: i) the total charged particles yields (or rapidity densities), which are taken from experiments at SPS [9,10] and RHIC [11] and extrapolated for LHC; and ii) $N_{c \bar{c}}^{d i}$, which is taken from next-to-leading order (NLO) perturbative QCD (pQCD) calculations for pp [12] (the yield from MRST HO parton distributions was used here) and scaled to AA via the nuclear overlap function. A constant temperature of $170 \mathrm{MeV}$ and the baryonic chemical potential $\mu_{b}$ according to parametrization $\mu_{b}=1270 /\left(1+\sqrt{s_{N N}} / 4.3\right)$ [13] have been used for the calculations.

\section{Results}

We first compare predictions of the model to $4 \pi$-integrated $J / \psi$ data at the SPS (NA50 data [14] replotted as outlined in [2]). The differences between this calculation and that of [2] are due to the inclusion in the present version of the complete set of charmed mesons (open and hidden) and baryons as well as due to updated values of the inputs (volume and open charm cross section) as outlined above. In Fig. 1 we present the comparison for two values of $N_{c \bar{c}}^{d i r}$ : from NLO calculations [12] and scaled up by a factor of 2.8. Note that the observed centrality dependence is well reproduced using the NLO cross sections for charm production scaled by the nuclear overlap function. To explain the overall magnitude of the data a $N_{c \bar{c}}^{d i r}$ increase by a factor of 2.8 compared to NLO calculations is needed.

We mention in this context that the observed enhancement of the dilepton yield at intermediate masses 15 has been interpreted as possible indication for an anomalous increase in the charm cross section. If, in our calculations, we scale-up $N_{c \bar{c}}^{d i r}$ from NLO according to the NA50 enhancement ("Data/Expected sources") as a function of $N_{\text {part }}$ [15], the $J / \psi$ data are underpredicted and the $N_{\text {part }}$ dependence is flatter (see Fig. 11). However, the NA50 suggestion of charm enhancement is controversial, and other explanations exist of the observed enhancement in terms of thermal radiation [16, 17.

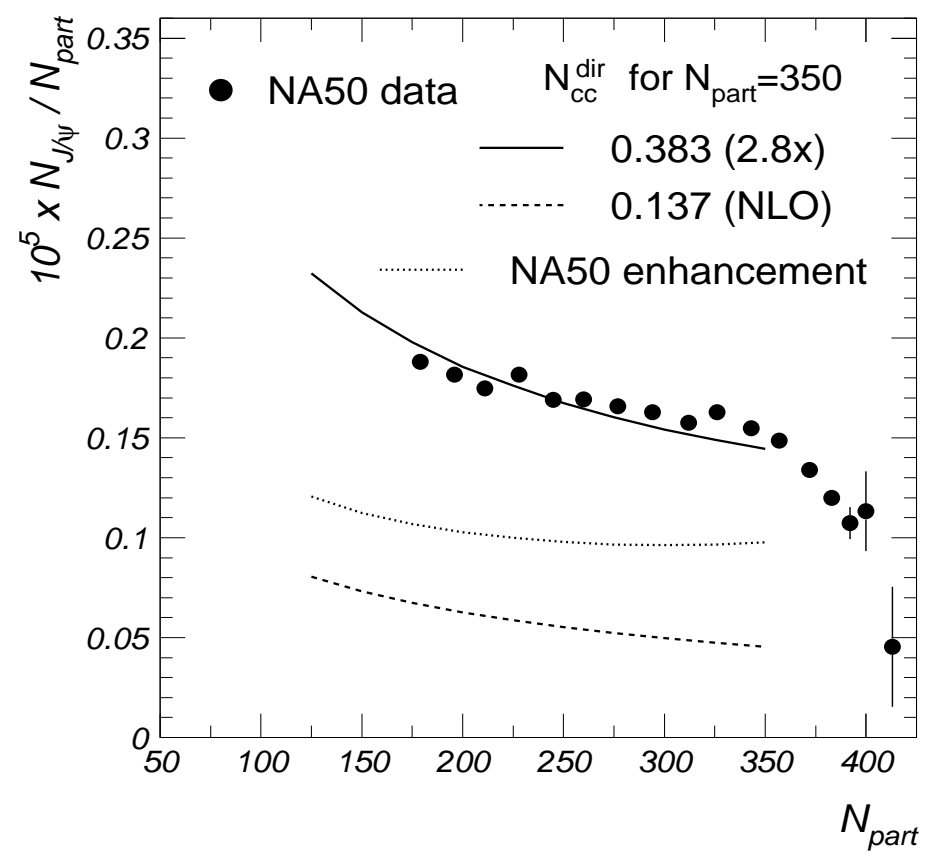

Figure 1. The centrality dependence of $J / \psi$ production at SPS. Model predictions are compared to NA50 data $(4 \pi-$ integrated). Two curves for the model correspond to values of $N_{c \bar{c}}^{\text {dir }}$ from NLO calculations [12] $\left(0.137\right.$, leading to $\left.g_{c}=0.78\right)$ and scaled up by a factor of 2.8 . The dotted curve is obtained when considering a charm enhancement as suggested by NA50 15]. For central collisions $\left(N_{\text {part }}=350\right)$ we use in the calculations $\mathrm{V}=3070 \mathrm{fm}^{3}$ and $N_{o c}^{t h}=0.98$. 
We turn now to predictions for collider energies. For comparison we include in this study also results at SPS energy. The input parameters for these calculations for central collisions $\left(N_{\text {part }}=350\right)$ are presented in Table 1. Notice that from now on we focus on rapidity densities, which are the relevant observables at the colliders. The results are compiled in Table 2 for a selection of hadrons with open and hidden charm. All predicted yields increase strongly with energy, reflecting the increasing charm cross section and the concomitant importance of statistical recombination. Also ratios of open charm hadrons evolve with increasing energy, reflecting the corresponding decrease in charm chemical potential.

Table 1

Input parameters for model calculations at SPS, RHIC and LHC.

\begin{tabular}{|c|ccc|}
\hline$\sqrt{s_{N N}}(\mathrm{GeV})$ & 17.3 & 200 & 5500 \\
\hline$T(\mathrm{MeV})$ & 170 & 170 & 170 \\
$\mu_{b}(\mathrm{MeV})$ & 253 & 27 & 1 \\
\hline $\mathrm{d} N_{c h} / \mathrm{d} y$ & 430 & 730 & 2000 \\
$V_{\Delta y=1}\left(\mathrm{fm}^{3}\right)$ & 861 & 1663 & 4564 \\
\hline $\mathrm{d} N_{c \bar{c}}^{d i r} / \mathrm{d} y$ & 0.064 & 1.92 & 16.8 \\
$g_{c}$ & 1.86 & 8.33 & 23.2 \\
\hline
\end{tabular}

\section{Table 2}

Results of model calculations at SPS, RHIC and LHC for $N_{\text {part }}=350$.

\begin{tabular}{|c|ccc|}
\hline$\sqrt{s_{N N}}(\mathrm{GeV})$ & 17.3 & 200 & 5500 \\
\hline $\mathrm{d} N_{\mathrm{D}^{+}} / \mathrm{d} y$ & 0.010 & 0.404 & 3.56 \\
$\mathrm{~d} N_{\mathrm{D}^{-}} / \mathrm{d} y$ & 0.016 & 0.420 & 3.53 \\
$\mathrm{~d} N_{\mathrm{D}^{0}} / \mathrm{d} y$ & 0.022 & 0.89 & 7.80 \\
$\mathrm{~d} N_{\Lambda_{c}} / \mathrm{d} y$ & 0.014 & 0.153 & 1.16 \\
$\mathrm{~d} N_{J / \psi} / \mathrm{d} y$ & $2.55 \cdot 10^{-4}$ & 0.011 & 0.226 \\
$\mathrm{~d} N_{\psi^{\prime}} / \mathrm{d} y$ & $0.95 \cdot 10^{-5}$ & $3.97 \cdot 10^{-4}$ & $8.46 \cdot 10^{-3}$ \\
\hline
\end{tabular}

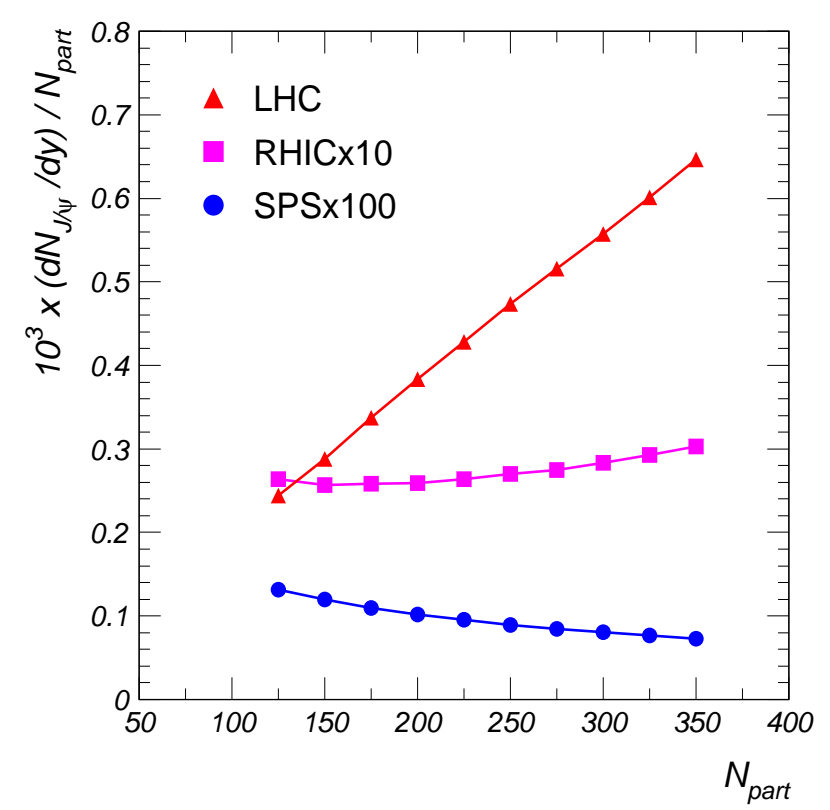

Figure 2. Centrality dependence of $J / \psi$ rapidity density at SPS, RHIC and LHC.

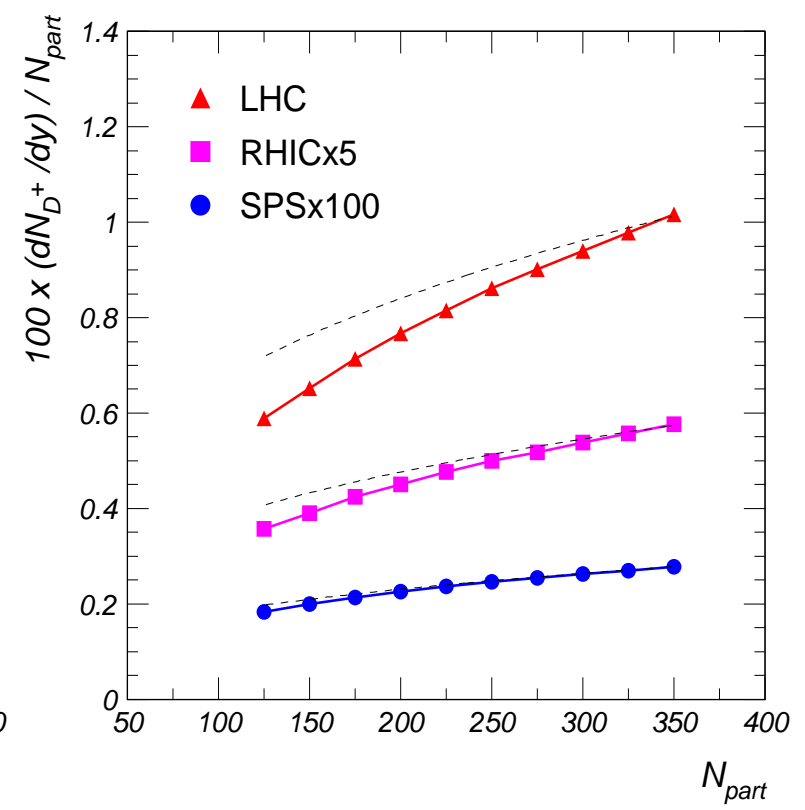

Figure 3. Centrality dependence of $\mathrm{D}^{+}$rapidity density at SPS, RHIC and LHC.

Predictions for the centrality dependence of $J / \psi$ production are presented in Fig. 2. In addition to the dramatic change in magnitude (note the scale-up by factors of 10 and 100 
for RHIC and SPS energy, respectively) the results exhibit a striking change in centrality dependence, reflecting the transition from a canonical to a grand-canonical regime (see [2] for more details). The preliminary PHENIX results on $J / \psi$ production at RHIC [18] agree, within the still large error bars, with our predictions. A stringent test of the present model can only be made when high statistics $J / \psi$ data are available. Another important issue in this respect is the accuracy of the charm cross section, for which so far only indirect measurements are available [19]. In any case, very large suppression factors as predicted, e.g., by [7] seem not supported by the data. In Fig. 3 we present the predicted centrality dependence of charged $\mathrm{D}^{+}$-meson production for the three energies. The expected approximate scaling of the ratio $\mathrm{D}^{+} / N_{\text {part }}$ like $N_{\text {part }}^{1 / 3}$ (dashed lines in Fig. (3) is only roughly fulfilled due to departures of the nuclear overlap function from the simple $N_{\text {part }}^{4 / 3}$ dependence.

\section{Conclusions}

We have demonstrated that the statistical coalescence approach yields a good description of the measured centrality dependence of $J / \psi$ production at SPS energy, albeit with a charm cross section increased by a factor of 2.8 compared to current NLO calculation. Rapidity densities for open and hidden charm mesons are predicted to increase strongly with energy, with striking changes in centrality dependence. First RHIC data on $J / \psi$ production support the current predictions, although the errors are too large to make firm conclusions. The statistical coalescence implies travel of charm quarks over significant distances in QGP. If the model predictions will describe consistently precision data this would be a clear signal for the presence of a deconfined phase.

\section{REFERENCES}

1. P. Braun-Munzinger and J. Stachel, Phys. Lett. B490 (2000) 196.

2. P. Braun-Munzinger and J. Stachel, Nucl. Phys. A690 (2001) 119c.

3. M. Gorenstein et al., Phys. Lett. B509 (2001) 277; hep-ph/0012292.

4. L. Grandchamp and R. Rapp, Phys. Lett. B523 (2001) 60; hep-ph/0205305.

5. R.L. Thews, M. Schroedter and J. Rafelski, Phys. Rev. C63 (2001) 054905.

6. T. Matsui and H. Satz, Phys. Lett. B178 (1986) 416.

7. R. Vogt (p. 250c) in S.A. Bass et al., Nucl. Phys. A661 (1999) 205c.

8. P. Braun-Munzinger and J. Stachel, J. Phys. G28 (2002) 1971.

9. NA49 collaboration, nucl-ex/0205002.

10. NA50 collaboration, Phys. Lett. B530 (2002) 33.

11. PHOBOS collaboration, Phys. Rev. Lett. 88 (2002) 022302.

12. R. Vogt, hep-ph/0203151; hep-ph/0111271.

13. P. Braun-Munzinger et al., Nucl. Phys. A697 (2002) 902.

14. NA50 collaboration, Phys. Lett. B450 (1999) 456; Phys. Lett. B477 (2000) 28.

15. NA50 collaboration, Nucl. Phys. A698 (2002) 539c.

16. R. Rapp and E. Shuryak, Phys. Lett. B473 (2000) 13.

17. K. Gallmeister, B. Kämpfer and O.P. Pavlenko, Phys. Lett. B473 (2000) 20.

18. J. Nagle, PHENIX collaboration, these proceedings.

19. R. Averbeck, PHENIX collaboration, these proceedings. 